\title{
From old Globular Clusters to early Structures in the Universe: the Formation of old, metal-poor Halos around early-type Galaxies
}

\author{
Markus Kissler-Patig \\ European Southern Observatory, Garching, Germany
}

December 1, 2001

\begin{abstract}
Old, metal-poor globular clusters trace the formation and evolution of early-type galaxies. Their are the best probes, at low redshift, of the building-up of galaxy halos at high redshift. Their properties constrain the characteristics of their progenitors. Recent results suggest that DLAs at $z>3$ are the likely hosts for their formation. Finally, they shed light on the old, metal-poor halos probably present around all early-type galaxies.
\end{abstract}

Keywords: early-type galaxy, halo, globular cluster

\section{Why old, metal-poor globular clusters?}

Old, metal-poor globular clusters are excellent tracers for the formation of galaxies (see recent reviews by Ashman \& Zepf 1998, Kissler-Patig 2000, Harris 2001). They are present in large numbers around all giant galaxies studied to date and can therefore be used as a common tool. They are old ( $\sim 12 \pm 2 \mathrm{Gyr})$ and thus witnessed the past since a time corresponding to a high redshift $(z>3$, somewhat dependent on the cosmology). They form as stars form, i.e. understanding their epochs of formation allows to study the star formation history of their host galaxy. Finally, they are simple stellar populations, easier to model and to understand than the diffuse stellar light of their host galaxies.

Globular cluster sub-populations turned out to be very common (Zepf \& Ashman 1993, and e.g. Gebhardt \& Kissler-Patig 1999). To first approximation, a typical globular cluster system shows two broad sub-populations. An old-metal poor sub-population is almost always present, as well as a more metal-rich (old to intermediate age, possibly itself divided into sub-populations). The properties of the latter resemble those of the diffuse stellar light. Indeed, the integrated light of earlytype galaxies appear to be dominated by the metal-rich component. Thus, the old, metal-poor globular clusters are a unique opportunity to study the halo component of early-type galaxies otherwise difficult to observe. At the same time, they allow to study (at low-redshift) the building up (at high redshift) of early-type galaxies. 


\section{The properties of the old, metal-poor globular cluster sub-populations}

Only since the mid-90s, studies investigate the properties of the various sub-populations of globular clusters around early-type galaxies, as opposed to focusing on the general properties of the entire system.

The first difference noticed (in NGC 4472) between the red and the blue sub-populations was their different density distributions (Geisler, Lee \& Kim 1996). The red clusters are significantly more concentrated towards the centre than the blue ones. This is found to be true in all other galaxies studied to date. Furthermore, the spatial distributions also appear to differ. E.g. in NGC 1380, an S0 galaxy, Kissler-Patig et al. (1997) found the red clusters to follow the ellipticity of the diffuse light while the blue clusters where spherically distributed. Finally, the kinematics of the two sub-populations were found to differ systematically in the studied cases (e.g. Kissler-Patig \& Gebhardt 1998, Zepf et al. 2000, Côté et al. 2001). In summary, the blue and the red globular clusters form two distinct sub-populations from their properties, and the blue (metal-poor) globular clusters have 'halo' properties.

The metal-poor globular cluster also stand out with respect to their sizes. While the sizes of clusters in nearby galaxies were all found to roughly correspond to the observed sizes in the Milky Way, the metal-poor clusters appear systematically larger than the metal-rich ones at all galacto-centric radii (e.g. Kundu \& Whitmore 1998, Puzia et al. 1999, Larsen et al. 2001). This result is interpreted as a imprint from the time of formation, hinting at the formation of metal-poor clusters in shallower potential wells, i.e. smaller fragments.

The abundances and abundance ratios of old, metal-poor clusters are best studied using spectroscopy of clusters in nearby galaxies. All old-metal poor clusters are found to lie in the same region not only in $\mathrm{H} \beta-\mathrm{MgFe}$ diagrams, but also in $\mathrm{Fe}-\mathrm{Mg}$ diagrams (e.g. KisslerPatig et al. 1998, Cohen et al. 1998, Schroder et al. 2001). This points to very similar ages and $\alpha$-element ratios among all old, metal-poor clusters in the nearby universe, including the Milky Way, M31 and M81.

Consequently, the mean metalicity of the metal-poor globular cluster sub-population in a given galaxy was found to be constant over a large range of galaxy sizes, morphologies and metalicity (e.g. Ashman \& Bird 1993, Burgarella et al. 2001). The properties of the metal-poor sub-populations correlate only weakly (if at all) with their host-galaxy properties. 
Finally, the globular cluster luminosity function was show to be a useful distance indicator, especially when using only old-metal clusters (e.g. Kissler-Patig 2000). This, in turn, proves that the mass distribution of the globular clusters is universal and independent of the galaxy properties: again suggesting a formation process largely unrelated to the final host galaxy.

The conclusion from the above is that old, metal-poor globular clusters are present in all observed early-type galaxies and have universal properties that do not depend (or only weakly) on the host galaxy properties. This suggests their formation in small fragments, largely independent of the final host galaxy.

\section{On the nature of their progenitor fragments}

The properties of old-metal poor globular clusters can constrain the nature of their formation sites. This was discussed by Burgarella, KisslerPatig \& Buat (2001), who identified damped lyman- $\alpha$ systems (DLAs) as likely sites for the formation of the old, metal-poor globular clusters.

Updating their result, the ages of old, metal-poor globular clusters (around $12 \pm 2 \mathrm{Gyr}$ ) correspond to a redshift of formation of $z=4_{-1.5}^{+\infty}$ in the current standard cosmology $\left(\Omega=0.3, \Lambda=0.7, \mathrm{H}_{0}=70\right.$ $\left.\mathrm{km} \cdot \mathrm{s}^{-1} \cdot \mathrm{Mpc}^{-1}\right)$. Comparing this with the latest abundance measurements of DLAs (e.g. Dessauges-Zavadsky et al. 2001), indicates that the mean abundance of DLAs at $z>3$ matches well the $\sim 1 / 50$ solar mean abundance of the old, metal-poor globular clusters. These DLAs are thus likely to be the fragments in which (one or more) globular clusters formed, while DLAs at lower redshift might, at least partly, belong to a different category of objects (more evolved spirals?).

From the properties of the old, metal-poor globular clusters we learn the following on the nature of the fragments:

- The fragments had metalicities between $-1.0<[\mathrm{Fe} / \mathrm{H}<-2.5$ dex, the range spanned by the old, metal-poor clusters.

- Their mass distribution is likely to have been a power-law of slope around -2 , transmitting this characteristic imprint to the mass function of the globular clusters (up to masses around $10^{8} \mathrm{M}_{\odot}$ ). The high mass cut-off as judged from the largest dwarf galaxies hosting metal-poor globular clusters only, must have lied around $10^{9}-10^{10} \mathrm{M}_{\odot}$.

- The fragments collapse at roughly $z>3$ without suffering the influence of the final host galaxies of which they formed the halos later. This way, they probably enriched significantly the intra-galactic medium. 


\section{Where are the old, metal-poor stars in ellipticals?}

Clearly, the presence of old, metal-poor globular cluster sub-populations calls for a stellar counter-part. Where are the stars associated with this halo population in early-type galaxies?

There is good evidence that the old, metal-poor globular clusters formed with a very high specific frequency $\left(\mathrm{S}_{N} \sim 20\right)$, i.e. have only few stars associated with them, while the metal-rich globular clusters formed together with the vast majority of the stars (low $S_{N} \sim 1-2$ ).

This is supported $i$ ) by the high specific frequency observed in dwarf galaxies and halos, dominated by old, metal-poor globular clusters, ii) by the relatively small amount of metal-poor stars contributing to the integrated light of early-type galaxies (see Maraston \& Thomas 2000, Lotz et al. 2000), iii) by the direct comparison of the metal-poor to metal-rich number ratios for stars and globular clusters in NGC 5128, the nearest giant elliptical (Harris, Harris \& Poole 1999): While the metal-rich stars vastly dominate in number the stellar population, metal-poor and metal-rich globular clusters appear in roughly similar numbers.

Therefore, we expect only a small population of old, metal-poor stars associated with the old, metal-poor globular clusters. Such a stellar population is difficult to detect within the diffuse stellar light dominated by the metal-rich stars.

\section{The formation of halos around early-type galaxies}

In summary: old, metal-poor globular clusters form an independent 'halo' sub-population present in all galaxies. Their properties suggest a formation in small fragments identified with DLAs at $z>3$. This implies that the metal-poor halos of giant ellipticals formed through the assembly of (mostly) collapsed small fragments. Their properties correlate only weakly if at all with the host galaxy properties, suggesting that some fragments might have been influence by the final host galaxy, but many (most?) formed as independent satellites.

\section{Acknowledgements}

Many thanks go to my collaborators D.Burgarella, V.Buat, C.Maraston and D.Thomas for fruitful discussions on old, metal-poor clusters, stellar populations and halos. 


\section{References}

Ashman, K.M., \& Bird, C.M. 1993, AJ 106, 2281

Ashman, K.M., \& Zepf, S.E. 1998, Globular Cluster Systems, Cambridge University Press

Burgarella, D., Kissler-Patig, M., \& Buat, V. 2001, AJ, 121, 2647

Cohen, J.G., Blakeslee, J.P., \& Ryzhov A. 1998, ApJ 486, 808

Côté, P., McLaughlin, D.E.; Hanes, D.A., et al. 2001, ApJ 559, 828

Dessauges-Zavadsky, M., D'Odorico, S., McMahon, R.G., Péroux, C. 2001, ASP conf.series Chemical Enrichment of the intra-cluster and inter-galactic Medium

Gebhardt, K. \& Kissler-Patig, M. 1999, AJ 118, 1526

Geisler, D., Lee, M.G., \& Kim, E. 1996 AJ 111, 1529

Harris, W.E., 2000, Star Clusters, Saas-Fee Advanced Course 28, Berlin, Heidelberg: Springer

Harris, G.L.H, Harris, W.E., \& Poole, G.B. 1999, AJ 117, 855

Kissler-Patig, M. 2000, Reviews in Modern Astronomy, Vol. 13, ed. Schielicke, 13

Kissler-Patig, M., Richter, T., Storm, J., Della Valle, M. 1997, A\&A 327, 503

Kissler-Patig, M., Brodie, J.P., Schroder, L.L. et al. 1998, AJ 115, 105

Kissler-Patig, M., \& Gebhardt, K. 1998, AJ 116, 2237

Kundu, A., \& Whitmore, B.C. 1998, AJ 116, 2841

Larsen, S.S., Brodie, J.P., Huchra, J.P. et al. 2001, AJ 121, 2974

Lotz, J.M., Ferguson, H.C., \& Bohlin, R.C. 2000, ApJ, 532, 830

Puzia, T.H., Kissler-Patig, M., Brodie, J.P., et al. 1999, AJ, 118, 2734

Maraston, C., \& Thomas, D. 2000, ApJ, 541, 126

Schroder, L.L., Brodie, J.P., Kissler-Patig, M., et al. 2001, AJ December issue

Zepf, S.E., \& Ashman, K.M. 1993, MNRAS, 264, 611

Zepf, S.E., Beasley, M.A.; Bridges, T.J., et al. , 2000, AJ 120, 2928 
kissler-patig.tex; 3/11/2018; 23:29; p.6 